\title{
Self-organizing traffic signal control with prioritization strategy aided by vehicular sensor network
}

\author{
Marcin Lewandowski, Bartłomiej Płaczek, and Marcin Bernas \\ University of Silesia, Institute of Computer Science, \\ Będzińska 39, 41-200 Sosnowiec, Poland \\ marcin.lewandowski@us.edu.pl, \{placzek. bartlomiej, marcin. bernas\}@gmail.com
}

\begin{abstract}
Preemption strategies are necessary for traffic signal control at intersections in a road network to ensure minimum delay of priority vehicles, such as ambulances or police cars. This paper introduces a decentralized algorithm, which extends the self-organizing signal control to provide preemption for the priority vehicles. The introduced algorithm enables effective utilisation of real-time data collected in vehicular sensor network (VSN). Results of simulation experiments show that the proposed approach ensures a quick passage of the priority vehicles and minimizes the negative effect of signal preemption on delays of non-priority vehicles. The new VSN-aided preemption strategy improves performance of the state-of-the-art methods that are based on road-side vehicle detectors and simple vehicle-to-infrastructure communication systems.
\end{abstract}

Keywords: vehicular sensor networks, self-organizing systems, traffic signal control, priority vehicles

\section{Introduction}

Effective traffic signal control is a key element of intelligent transport systems, which improves utilization of the existing road infrastructure and increases its capacity [1]. State-of-the-art traffic control approaches are based on centralized techniques that require global information about traffic conditions in the considered road network. The centralized traffic control methods are computationally complex and inherently non-scalable. Low scalability of the centralized control algorithms has motivated the current interest in decentralized self-organizing traffic control $[2,3]$. The self-organizing traffic signals are controlled independently for each intersection in the network on the basis of real-time traffic information obtained from local measurements. The information required by self-organizing traffic control describes current traffic conditions at road segments connected to a particular intersection.

Recent advances in vehicular sensor networks (VSNs) facilitate wireless data transfer between vehicles and infrastructure [4-6]. The vehicles in VSN can collect detailed and useful information regarding their current positions and velocities. The collected data can be then transmitted via wireless communication to 
a control unit at the nearest intersection. This technology enables cost-effective collection of the local traffic information in real-time. The increased availability of detailed real-time traffic information results in high application potential of the VSN-aided self-organizing traffic control.

This paper focuses on performance analysis of the self-organizing traffic control with a preemption strategy. The aim of signal preemption strategies is to efficiently clear the paths for priority vehicles, such as ambulances, police cars, fire engines, transit buses, light rail vehicles, snow ploughs, etc [7-9]. Preemption interrupts normal signal operations to serve the priority vehicle as soon as possible with little or no delay [10]. An algorithm is proposed in this paper, which extends the self-organizing signal control to provide preemption for priority vehicles. The objective of the proposed algorithm is to ensure a quick passage of the priority vehicles and minimize the negative effect of signal preemption on delays of non-priority vehicles.

Performance of the proposed algorithm was examined in a simulation environment with application of different self-organizing control strategies from the literature. Two scenarios for the preemption strategy were considered during the simulation experiments. In the first scenario a priority vehicle sends preemption request to the traffic control unit as it approaches the intersection and the control unit responds immediately by switching traffic lights. This scenario corresponds to the current solutions that utilize the vehicle-to-infrastructure (V2I) communication $[11,12]$. The new approach with VSN-aided preemption is considered in the second scenario. In this case, the traffic lights are not switched immediately, when the request from priority vehicle is received. Instead, the traffic control unit evaluates time of preemption operation, which allows the priority vehicle to pass the intersection without stopping. This evaluation is based on real-time information about vehicles position delivered from VSN.

The paper is organized as follows. Related works are discussed in Section 2. Section 3 introduces the proposed method. Results of simulation experiments are presented in Section 4. Finally, conclusions are given in Section 5.

\section{Related works}

Several decentralized control approaches for the self-organizing traffic signals have been proposed in the related literature. An advantage of the self-organizing methods discussed in this section is high scalability, which is ensured as these methods do not require any central controller and do not involve communication between local control units at intersections.

A simple algorithm for self-organizing traffic lights (SOTL) was presented in [13]. According to this algorithm, a preference is given to vehicles that have been waiting longer, and to larger groups of vehicles (platoons). The experimental results reported in [14] show that SOTL can achieve higher performance and increase the network capacity in comparison to the conventional Sydney Coordinated Adaptive Traffic System. 
A more sophisticated self-organizing traffic control method was introduced by Lämmer and Helbing [15]. This method takes into account priorities that correspond to expected number of vehicles entering the intersection during a given time horizon. The expected numbers of vehicles are determined in this method by short-term traffic flow prediction based on a macroscopic fluid-dynamic model. Experimental comparison of this method with conventional adaptive control in a real-world road network revealed higher effectiveness of the self-organizing approach. Another work along these lines [16] introduces a self-organizing traffic signal system, where an interval microscopic traffic model is used to predict effects of possible control actions in a short time horizon.

Back-pressure [17] is a control strategy for the self-organizing traffic signals, which takes into account differences in traffic load on the road leading into the intersection and those leading out. The back-pressure method was originally proposed for routing algorithms in wireless networks as a decentralized scheme, which can provide maximum network throughput under the assumptions that all links in the network have infinite capacities. This concept was then adapted to urban road networks for signal control.

In [18] a cellular automata model of self-organizing traffic signal system was proposed, which enables evolutionary optimization of control rules that rely on adaptation to local variations in traffic state and enable effective coordination of the vehicular traffic at a network level. Fitness function, which guides the evolution of control rules, is evaluated in this approach via traffic simulation by using a microscopic cellular automata model.

A physic-based self-organizing control scheme for traffic signals was proposed in [2]. This method uses "virtual impulses" given by red signals or preceding cars, which are defined in a similar manner as the impulses used in physics. The virtual impulses are calculated at each traffic signal by using an optimal velocity model. They characterize to what extent vehicles will slow down owing to the existence of the preceding cars or red signals. The traffic signals are switched to reduce these virtual impulses.

A considerable research has also been devoted to signal preemption strategies that utilize V2I communication. Such strategies allow priority vehicles to send green light requests to traffic control units when approaching signalized intersections. The ZigBee communication was used in [19] for emergency vehicle clearance. According to that simple solution, the control unit at an intersection switches the traffic signals immediately, when it receives a preemption request from the emergency vehicle. The traffic lights are switched back to the normal operation once the priority vehicle passes through, and the control unit no longer receives the ZigBee signal.

Another simple preemption strategy was studied in [11] with application of the WAVE standard (IEEE 802.11p). According to this method the distance between the emergency car and the traffic lights is calculated in discrete time steps. If the distance is less than the specified value (which is defined in advance), the traffic light status changes to green for the emergency vehicle and red for the 
other cars. After the emergency vehicle passes the traffic light (with a distance of 20 meters) the traffic light status returns to its original state.

In [20] a signal control strategy was proposed, which decreases response time of emergency vehicles by employing V2I communication and a beaconing concept based on the WAVE standard as well as the prediction of queue length. According to that strategy, the traffic signals are adjusted adaptively to provide an early green at the right time so that the queue at the downstream intersections can be served just in time for the arrival of an emergency vehicle.

When using the V2I communication, it is possible that multiple priority vehicles will send requests such that there may be multiple active requests at the same time. An optimisation problem was formulated in [21] to explicitly accommodate the multiple priority requests from different modes of vehicles while simultaneously considering signal coordination and vehicle actuation.

In this paper a decentralized algorithm is proposed, which combines the selforganizing signal control with a preemption strategy. The introduced algorithm enables effective utilisation of real-time position and speed data collected from particular vehicles in VSN.

\section{Proposed approach}

This section introduces an algorithm, which allows the self-organizing signal control to provide preemption for priority vehicles. The objective of the preemption procedure is to serve the priority vehicles as soon as possible with minimum negative impact on the delay of non-priority vehicles. To this end, the proposed procedure utilizes data collected in VSN. The considered VSN is composed of vehicle nodes and control units that take control decisions and manage traffic signals at intersections by using the self-organizing strategy. Vehicles are equipped with sensors that collect speed and position data. The collected information is periodically transmitted from vehicles to control units.

Pseudocode of the self-organizing traffic signal control algorithm with preemption is presented in Algorithm 1. The input data of this algorithm consists of parameters that describe the traffic streams passing through an intersection. Output of the algorithm is a control decision that determines which traffic lane (or lanes) should get a green signal for a subsequent time interval. The consecutive control decisions are taken in constant time steps. In order to take control decision, priorities are assigned to the traffic lanes approaching the intersection. The priorities for traffic lanes are calculated dynamically based on current traffic data delivered from VSN.

According to the proposed algorithm, a preemption_condition function is used to verify if a priority vehicle for which the preemption has to be executed immediately is present in given traffic lane (line 5 of the pseudocode). The preemption condition takes into account expected time in which the priority vehicle will reach the traffic signal $\left(t_{R}\right)$ and the predicted time, which is necessary to clear the queue of vehicles in the considered lane $\left(t_{C}\right)$. This condition is defined as follows: 


$$
t_{R} \leqslant t_{G}+t_{C},
$$

where $t_{G}$ is required intergreen time between the green period terminating for traffic lanes that are losing right of way and the start of the green period for the lane where priority vehicle is detected. The intergreen time has to be introduced to ensure traffic safety. Signal reaching time is calculated as:

$$
t_{R}=d / v_{F},
$$

where $d$ denotes distance of the priority vehicle to traffic signal and $v_{F}$ is desired speed of the priority vehicle, which is observed in low traffic (free flow) conditions and in the absence of traffic signals. The vehicle node calculates the desired speed based on collected historical sensor readings and reports it to the control unit. Queue clearance time is estimated by using the formula:

$$
t_{C}=n / s+t_{0}
$$

where $n$ denotes number of vehicles in a queue ahead of the priority vehicle, $s$ is saturation flow rate, i.e. the maximum number of vehicles that can pass through the intersection during a time unit, when the traffic signal is green, $t_{0}$ is the time lost at the beginning of green light, when vehicles are accelerating.

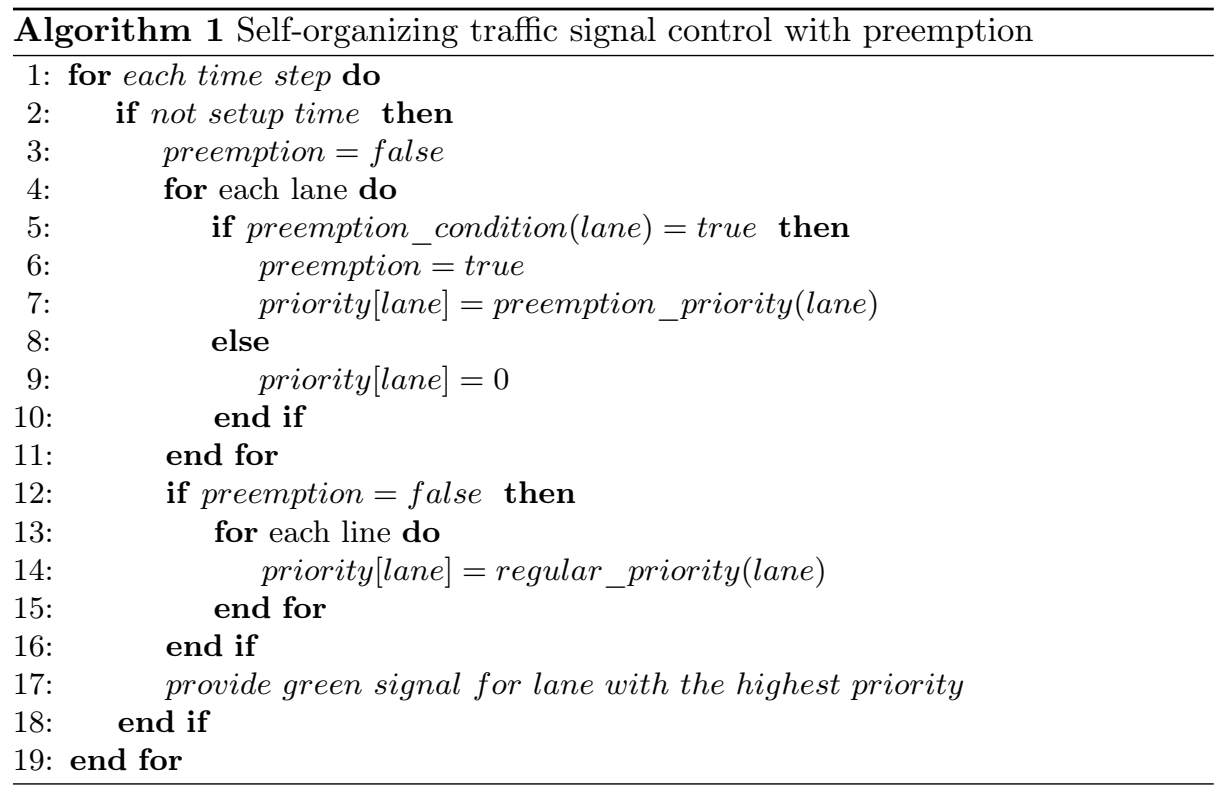

If preemption condition is satisfied for a given lane, then a priority of the preemption is calculated using the preemption_priority function. The preemption 
priority is useful for taking control decision when priority vehicles are approaching in two or more conflicting traffic lanes (that cannot get the right of way in the same time). This priority is calculated as follows:

$$
\text { preemption_priority }=\frac{m}{t_{G}^{\text {start }}+t_{S}+t_{G}^{\text {end }}},
$$

where $m$ is number of priority vehicles in the given lane, $t_{G}^{\text {start }}$ is intergreen time, which has to be introduced before giving green signal for the considered lane $\left(t_{G}^{\text {start }}=0\right.$ if the considered lane already has the green signal $), t_{S}$ is the green time necessary for the $m$ priority vehicles to leave the intersection, and $t_{G}^{e n d}$ denotes intergreen time after green signal for the considered lane. The above definition of the preemption priority is based on the approach presented in [15].

If the preemption condition is not satisfied for all traffic lanes, then the proposed algorithm uses the regular_priority function to calculate the priority of each lane in accordance with a selected state-of-the-art traffic control method. The regular_priority function also assures that all traffic lanes at the intersection will get the green signal at least once in a predetermined time period. In this study different definitions of the regular_priority function are taken into consideration, as discussed in the next section. These definitions correspond to various self-organizing traffic control methods from the literature.

During setup time, which includes the intergreen time and a minimum green time, the priority computation and decision making procedure is skipped because the service cannot be switched to another traffic lane.

\section{Experiments}

Simulation experiments were performed to compare effectiveness of the proposed VSN-aided preemption strategy with the state-of-the-art methods. Application of various self-organizing traffic control methods was considered in this study. The experimental results presented in this section concern delay and average speed of both the priority and non-priority vehicles. Based on these results, impact of priority vehicles on the performance of different self-organizing traffic control strategies is analysed.

\subsection{Simulation setup}

In this study the simulation experiments were conducted in SUMO (Simulation of Urban MObility) [19]. SUMO is a widely recognized open-source traffic simulation package including a traffic simulator as well as supporting tools. The simulator is microscopic, space-continuous and time-discrete, providing a fair approximation of real world traffic scenarios. Topology of the simulated network is a lattice of 8 bidirectional roads with 16 signalized intersections. Schema of the simulated road network and an intersection example are presented in Fig. 1. During single run of simulation, the traffic intensity was changed from 0.02 to 0.14 vehicles per second. The vehicles were generated with the same intensity 
at each entrance of the road network. One simulation run corresponds to one hour. Percentage of the priority vehicles was changed between $0 \%$ and $3.5 \%$. This percentage was determined for each one-hour simulation run. Two types of the priority vehicles were considered: slow, with maximum speed of $60 \mathrm{~km} / \mathrm{h}$, and fast, with the maximum speed of $72 \mathrm{~km} / \mathrm{h}$. Maximum speed of the non-priority vehicles was equal to $60 \mathrm{~km} / \mathrm{h}$. Results of the simulation include stop delays and average speeds of vehicles. The results were collected for four intersections in centre of the simulated road network.

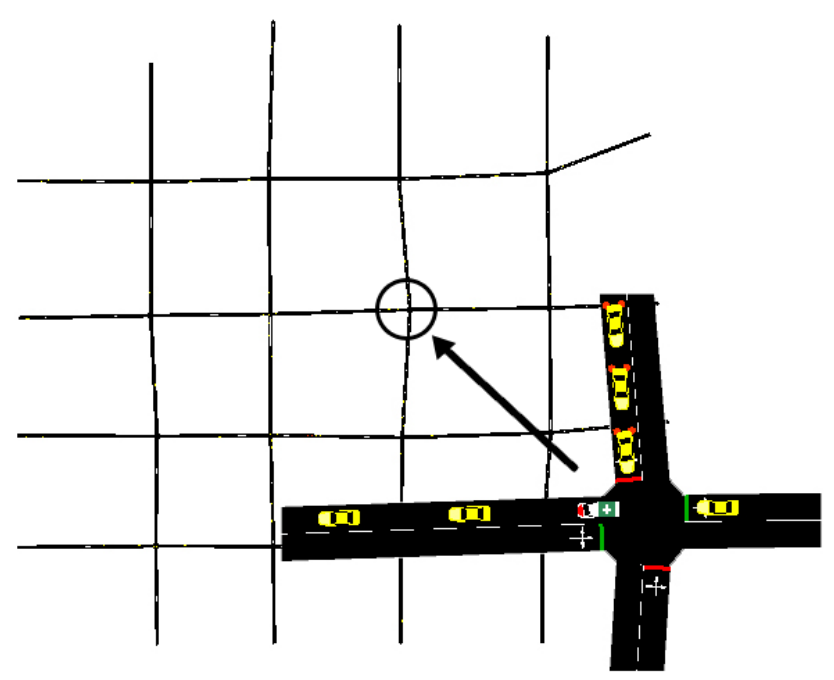

Fig. 1. Simulated road network.

Traffic signals at the simulated intersections were controlled by using decentralized self-organizing algorithms. Six different signal control algorithms were implemented in the simulation environment (Tab. 1). These algorithms use three control strategies (SOTL, LH, and BP) to calculate the regular priorities for traffic lanes, when priority vehicles are absent.

The SOTL (Self-Organizing Traffic Lights) strategy [15] takes into account current counts of vehicles approaching an intersection. Each traffic signal has a counter, which is set to zero when the signal turns red and then it is incremented at each time step by the number of vehicles approaching this red signal. When the counter reaches a predetermined threshold, the traffic lane gets the highest priority. In order to prevent the traffic signals from switching too frequently, the minimum green time constraint is used. Another constraint in the SOTL strategy keeps the small groups of vehicles (platoons) together and allows for dividing the large platoons that would excessively block the traffic flow of intersecting streets. Pseudo-code of the SOTL strategy can be found in [13]. 
Table 1. Compared algorithms

\begin{tabular}{|c|c|c|c|c|c|c|}
\hline Algorithm & SOTL1 & LH1 & BP1 & SOTL2 & LH2 & BP2 \\
\hline $\begin{array}{c}\text { Source of information } \\
\text { about non-priority } \\
\text { vehicles }\end{array}$ & RSD & VSN & Road-side detectors (RSD) & VSN & RSD \\
\hline $\begin{array}{c}\text { Source of information } \\
\text { about priority vehicles }\end{array}$ & RSD or V2I communication & \multicolumn{2}{|c|}{ VSN } \\
\hline Preemption condition & \multicolumn{3}{|c|}{ Distance to signal } & Time to reach signal \\
\hline Preemption priority & \multicolumn{3}{|c|}{ Order of requests } & \multicolumn{2}{c|}{ Minimum delay } \\
\hline
\end{tabular}

In case of LH strategy [16], which is based on the method introduced by Lämmer and Helbing, two control rules are used: optimization rule, and stabilisation rule. According to the optimization rule, a cost associated with providing green signal for a particular lane is predicted as a total increase of vehicle delay. The prediction is performed by using a microscopic traffic model, which is based on cellular automata. The traffic lane with minimum cost gets the highest priority. The objective of the stabilisation is to assure that all traffic lanes will get a green signal at least once in a maximum time period. More detailed information about the optimization and stabilisation rules can be found in [15].

The idea of BackPressure (BP) strategy [17] is to compute pressures at every intersection of the network based on queue lengths and to give priority for traffic lanes with a high upstream pressure and a low downstream pressure. This strategy uses priorities that are proportional to the difference of queue lengths in traffic lanes leading into the intersection and those leading out. Pseudo-code of the BP strategy can be found in [17].

The LH strategy uses a microscopic cellular automata model to map current positions and velocities of particular vehicles. Thus, this strategy can fully utilize the detailed data from VSN. In contrast, the SOTL and BP strategies are designed for cooperation with state-of-the-art road-side detectors that deliver information about vehicle counts and queue lengths in traffic lanes.

The signal control algorithms in Tab. 1 are categorized into two groups with regard to the preemption method. For the first group (SOTL1, LH1, and BP1) the state-of-the-art preemption method is used, which means that the preemption request is registered when the distance of priority vehicle to intersection is below a predetermined threshold. In case of many requests, the highest priority is assigned to the first registered request. This scenario corresponds to the current solutions that utilize the V2I communication or the road-side detectors. Utilization of the detailed VSN data (i.e., current position and speed of the priority vehicles) for preemption purposes is possible for the second group of algorithms (SOTL2, LH2, and BP2). These algorithms include the preemption condition and the preemption priority functions that were proposed in Sect. 3. 


\subsection{Experimental results}

A signal preemption strategy should enable a quick passage of the priority vehicles with a minimum negative effect for the non-priority vehicles. The traffic simulations were conducted to evaluate average speed and stop delay of vehicles for the compared algorithms. In this section the simulation results are analysed to determine the impact of signal preemption on speed and delay of the non-priority vehicles.

Average delay and speed of non-priority vehicles for the considered algorithms are compared in Fig. 2. The lowest delay and the highest speed were observed for the LH algorithm, which utilizes the detailed data delivered from VSN. The algorithms designed for the state-of-the-art road-side detectors (SOTL and BP) have achieved a lower performance. When comparing the algorithms that employ the state-of -the-art preemption strategy (denoted by the suffix 1) with those implementing the proposed VSN-aided signal preemption (denoted by the suffix 2 ), it can be observed that the proposed approach ensures lower delay and increased speed of the vehicles. The introduced preemption strategy has decreased the average delay by $11 \%$ for algorithm LH, $6 \%$ for algorithm SOTL, and $2 \%$ for algorithm BP (Fig. 2 a). Moreover, the average speed was increased by $2 \%$ for $\mathrm{LH}$ and $1 \%$ for SOTL. In case of algorithm BP, the average speed remains at the same level (Fig. 2 b).

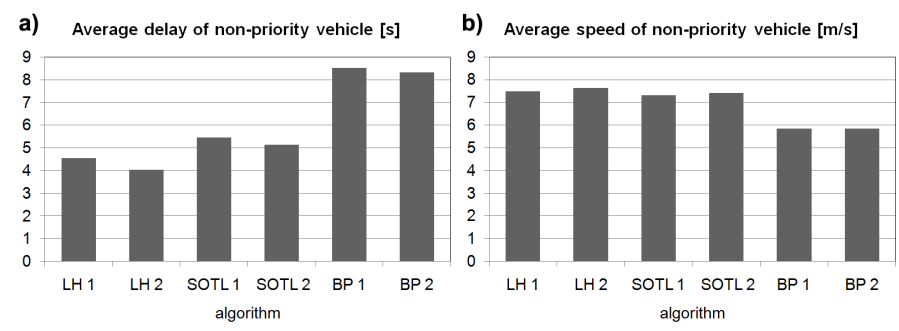

Fig. 2. Average delay and average speed of non-priority vehicles for the compared algorithms.

The chart in Fig. 3 compares average speed of priority vehicles for the considered algorithms. These results show that the proposed VSN-aided preemption strategy does not decrease the average speed of priority vehicles for the LH algorithm. It means that the LH2 algorithm, which utilizes the data collected from VSN to the largest extent, allows the delay of non-priority cars to be reduced and at the same time ensures high speed of the priority vehicles. In case of SOTL and BP algorithms the reduced delay of non-priority cars is obtained at the cost of lower speed of the priority vehicles.

Detailed results of the simulation for LH and SOTL algorithms are presented in Figs. 4 and 5. Each data point in these charts corresponds to results of onehour traffic simulation. For particular runs of the simulation, the model settings 


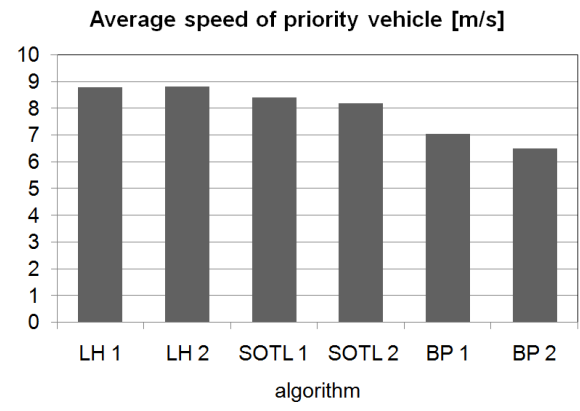

Fig. 3. Average speed of priority vehicles for the compared algorithms.

were adjusted in order to obtain different percentages of the priority vehicles. The results show that average delay of non-priority vehicles increases with the percentage of the priority vehicles. In contrary, average speed of non-priority cars decreases as the percentage of priority vehicles increases. Such dependencies have been observed for all analysed algorithms.
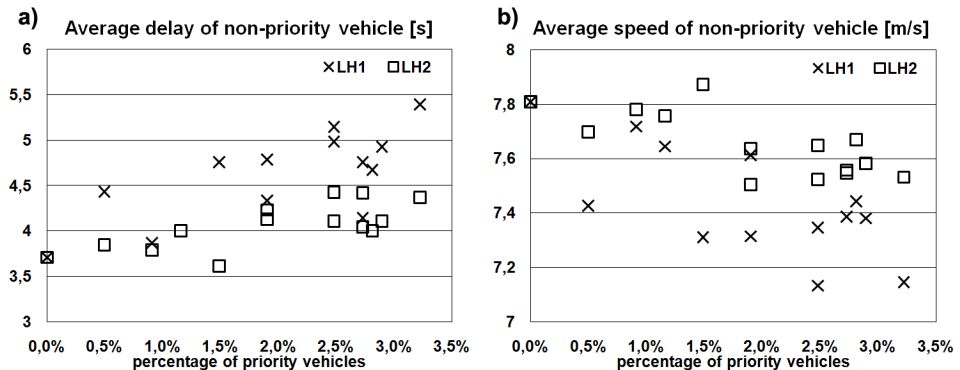

Fig. 4. Average delay and average speed of non-priority vehicles for the LH algorithms.

Comparison of the results obtained for algorithm LH1 with those of algorithm LH2 (Fig. 4) confirms that the VSN-aided preemption strategy decreases the negative impact of priority vehicles on delay and speed of the non-priority vehicles. This effect can be observed for a wide range of percentages of priority vehicles. Similar observations are made when comparing the results of algorithms SOTL1 and SOTL2 (Fig. 5). The better results for the proposed preemption strategy (LH2 and SOTL2) are related to the fact that the required time of preemption operation, which allows the priority vehicle to pass the intersection without stopping, can be accurately evaluated by using the detailed data from VSN. Thus, the normal signal operations are interrupted for a shorter time than in case of the state-of-the-art preemption method, which takes into account only the distance of priority vehicle to the traffic signal. 

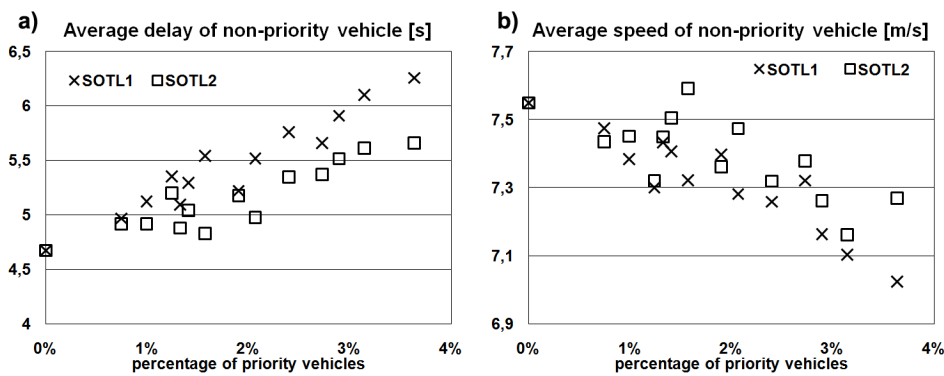

Fig. 5. Average delay and average speed of non-priority vehicles for the SOTL algorithms.

\section{Conclusion}

The introduced traffic control algorithm with preemption strategy enables effective utilisation of the data collected from VSN, which describe positions and velocities of particular vehicles. These data allow the control unit to accurately decide when a signal preemption operation has to be executed in order to clear the path through intersection for a priority vehicle. As a consequence, the negative impact of signal preemption on the flow of non-priority vehicles can be minimized. Results of simulations show that the proposed VSN-aided preemption strategy improves performance of the state-of-the-art methods that were designed for cooperation with road-side vehicle detectors or simple V2I communication solutions. The proposed preemption strategy was combined with the self-organizing signal control method. Thus, the proposed algorithm is scalable and can be implemented in decentralized systems, where the traffic signals are controlled independently for a large number of intersections. Moreover, the input data for this algorithm have to be collected locally from vehicles at road segments connected directly to a particular intersection. It means that the cost of data collection in VSN can be kept at a low level. Further research is necessary to test the proposed approach in different scenarios with multimodal priority.

\section{References}

1. Celiński, I., Sierpiński, G.: Traffic signal control system with extended logic in the context of the modal split. IERI Procedia, 4, 148-154 (2013).

2. Kano, T., Sugiyama, Y. and Ishiguro, A.: Autonomous Decentralized Control of Traffic Signals that can Adapt to Changes in Traffic. Collective Dynamics, 1(A5), 1-18 (2016).

3. Płaczek, B.: A Cellular Automata Approach for Simulation-Based Evolutionary Optimization of Self-Organizing Traffic Signal Control. Journal of Cellular Automata, 11(5-6), 475-496 (2016).

4. Younes, M. B., Boukerche A.: Intelligent Traffic Light Controlling Algorithms Using Vehicular Networks. IEEE Transactions on Vehicular Technology, 65(8), 5887-5899 (2016). 
5. Płaczek, B.: Selective data collection in vehicular networks for traffic control applications. Data Management in Vehicular Networks, 23, 14-28 (2012).

6. Płaczek, B., Bernas, M.: Optimizing Data Collection for Object Tracking in Wireless Sensor Networks, Communications in Computer and Information Science, Vol. 370, pp. 485-494, (2013).

7. Nellore, K., Hancke, G. P.: Traffic Management for Emergency Vehicle Priority Based on Visual Sensing, Sensors 16(11), 1892 (2016).

8. Zhanga, Z., Hea Q., Gouc J., Lid X.: Performance Measure for Reliable Travel Time of Emergency Vehicles. Transportation Research Part C: Emerging Technologies, 65, 97-110 (2016).

9. Yang M., Wang W., Han J.: Performance of the Priority Control Strategies for Bus Rapid Transit: Comparative Study from Scenario Microsimulation Using VISSIM. Discrete Dynamics in Nature and Society, 2013, (2013).

10. Zamanipour, M., Head, K. L., Feng, Y.: Efficient Priority Control Model for Multimodal Traffic Signals. Transportation Research Record: Journal of the Transportation Research Board, 2557, (2016).

11. Noori, H.: Impact of VANET-Based Traffic Signal Control on the Response Time of Emergency Vehicles in Realistic Large Scale Urban Area. IEEE International Conference on Communication Workshop, (2013).

12. Bernas, M.: VANETs as a Part of Weather Warning Systems, Communications in Computer and Information Science, Vol. 291, pp. 459-466, (2012).

13. Cools, S. B., Gershenson, C., D?Hooghe, B.: Self-organizing traffic lights: A realistic simulation in: Advances in Applied Self-Organizing Systems. Springer, 45-55 (2013).

14. Zhang, L., Garoni, T. M., de Gier, J.: A comparative study of Macroscopic Fundamental Diagrams of arterial road networks governed by adaptive traffic signal systems. Transportation Research Part B: Methodological, 49, 1-23 (2013).

15. Lämmer, S., Helbing, D.: Self-control of traffic lights and vehicle flows in urban road networks. Journal of Statistical Mechanics: Theory and Experiment, 2008(4), 4-19 (2008).

16. Płaczek, B.: A self-organizing system for urban traffic control based on predictive interval microscopic model. Engineering Applications of Artificial Intelligence, 34, 75-84 (2014).

17. Wongpiromsarn, T., Uthaicharoenpong T., Wang Y., Frazzoli E., Wang D.: Distributed traffic signal control for maximum network throughput. In: 15th International IEEE Conference on Intelligent Transportation Systems, ITSC 2012, pp. 588-595 IEEE (2012).

18. Placzek, B.: A Cellular Automata Approach for Simulation-Based Evolutionary Optimization of Self-Organizing Traffic Signal Control. Journal of Cellular Automata, 11(5/6), 475-496 (2016).

19. Sundar, R., Hebbar S., Golla, V.: Implementing Intelligent Traffic Control System for Congestion Control, Ambulance Clearance, and Stolen Vehicle Detection. IEEE Sensors Journal, 15(2), 1109-1113 (2015).

20. Noori, H., Fu, L., Shiravi, S.: A Connected Vehicle Based Traffic Signal Control Strategy for Emergency Vehicle Preemption. In: 2016 95th Transportation Research Board, 16-6763 (2016).

21. He, Q., Head, K. L., Ding, J.: Multi-modal traffic signal control with priority, signal actuation and coordination. Transportation Research Part C: Emerging Technologies, 46, 65-82 (2014).

22. Behrisch, M., Bieker, L., Erdmann, J., Krajzewicz, D.: SUMO simulation of urban mobility: an overview. In Proceedings of SIMUL 2011, The Third International Conference on Advances in Syston. ThinkMind. (2011). 